\title{
Inducing a many-body topological state of matter through Coulomb-engineered local interactions
}

\author{
M. Rösner $\odot^{1, *}$ and J. L. Lado $\odot^{2, \dagger}$ \\ ${ }^{1}$ Radboud University, Institute for Molecules and Materials, NL-6525 AJ Nijmegen, The Netherlands \\ ${ }^{2}$ Department of Applied Physics, Aalto University, 00076 Aalto, Espoo, Finland
}

(Received 17 September 2020; accepted 2 March 2021; published 19 March 2021)

\begin{abstract}
The engineering of artificial systems hosting topological excitations is at the heart of current condensed matter research. Most of these efforts focus on single-particle properties, neglecting possible engineering routes via the modifications of the fundamental many-body interactions. Interestingly, recent experimental breakthroughs have shown that Coulomb interactions can be efficiently controlled by substrate screening engineering. Inspired by this success, we propose a simple platform in which topologically nontrivial many-body excitations emerge solely from dielectrically engineered Coulomb interactions in an otherwise topologically trivial single-particle band structure. Furthermore, by performing a realistic microscopic modeling of screening engineering, we demonstrate how our proposal can be realized in one-dimensional systems such as quantum-dot chains. Our results put forward Coulomb engineering as a powerful tool to create topological excitations, with potential applications in a variety of solid-state platforms.
\end{abstract}

DOI: 10.1103/PhysRevResearch.3.013265

\section{INTRODUCTION}

Topology represents one of the most fertile fields in modern condensed matter physics [1-3], boosted by the prediction and experimental realization of topological systems, ranging from quantum spin Hall [4] and Chern [5,6] insulators to topological superconductors [7-9] and topological crystalline insulators $[10,11]$. Besides being of fundamental interest, these states are widely discussed due to their potential impact on solid-state technology, including low-consumption electronics [12], spintronics [13], and topological quantum computing [14]. The topological classification of noninteracting systems continues to grow with the recent examples of higher-order topological insulators $[15,16]$, non-Hermitian topology [17-19], fragile topological phases [20,21], quasiperiodic topology [22,23], and random topological systems [24,25].

Some of the most groundbreaking discoveries in condensed matter physics have been intimately related with strong interactions, with the paradigmatic examples of hightemperature superconductivity [26] and fractional quantum Hall physics $[27,28]$. It is thus not surprising that the interplay of topology and correlation effects is increasing as one of the most enigmatic fields in modern condensed matter physics. Topological states associated with interactions in topological Mott [29] and Kondo [30,31] insulators represent examples.

\footnotetext{
*m.roesner@science.ru.nl

†jose.1ado@aalto.fi

Published by the American Physical Society under the terms of the Creative Commons Attribution 4.0 International license. Further distribution of this work must maintain attribution to the author(s) and the published article's title, journal citation, and DOI.
}

In many instances, the role of interactions in generating topology is, however, reduced to a mean-field single-particle effect [29-32]. The potentially genuine role of interactions in topological systems beyond mean-field single-particle effects is, hence, still at an early stage.

Here, we demonstrate that Coulomb-engineered local interactions allow us to induce many-body topological excitations. Our mechanism puts forward a paradigm to generate topological states purely driven by electronic interactions. These topological states result from imprinting quasiperiodic structures to the local Coulomb interactions $U_{n}$ only, without requiring modifications to the topologically trivial singleparticle dispersions. Crucially, we show how such a spatial structuring of the local interactions can be experimentally achieved using quantum-dot (QD) arrays and by exploiting Coulomb engineering [33-36] via structured substrate, as depicted in Figs. 1(a) and 1(b). This paper is organized as follows: In Sec. II, we present how spatially modulated Coulomb interactions give rise to topological modes at the mean-field level. In Sec. III, we show how topological modes appear from these Coulomb interactions in a purely manybody model without a single-particle analog. In Sec. IV, we show how modulated Coulomb interactions can be realized by dielectric engineering. Finally, in Sec. V, we summarize our conclusions.

\section{SCREENING-INDUCED SINGLE- PARTICLE TOPOLOGY}

To exemplify our proposal using a simple Hamiltonian, we start with a model in which interactions generate a topological state that can be understood on the single-particle level within a mean-field framework. We consider a linear array of QDs with a single level per dot and spatially modulated local Coulomb interactions. The corresponding Hamiltonian 
(a)
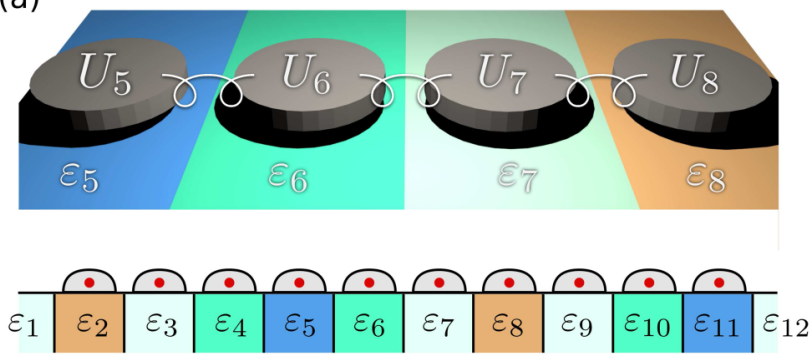

(b)

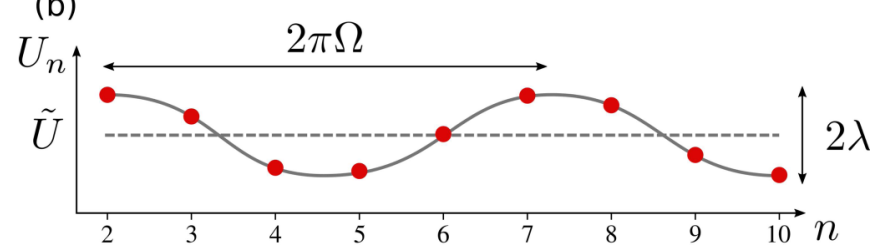

FIG. 1. (a) Sketch of the proposed system: A quantum-dot (QD) chain is deposited on a structured substrate. The spatial modulation of the substrate permitivity results in a spatially dependent environmental screening resulting in site-dependent local Coulomb interactions (b) within the otherwise homogeneous QD chain.

reads

$$
H=\sum_{n, s} t c_{n, s}^{\dagger} c_{n+1, s}+\sum_{n} U_{n} c_{n, \uparrow}^{\dagger} c_{n, \uparrow} c_{n, \downarrow}^{\dagger} c_{n, \downarrow}+\text { H.c., }
$$

where $n$ is the QD site index, $t$ the nearest-neighbor hopping, and $U_{n}$ a spatially varying local Coulomb repulsion of the form $U_{n}=\tilde{U}[1+\lambda \cos (\Omega n+\phi)]$, which is periodically modulated around a constant $\tilde{U}$. Here, $\Omega, \phi$, and $\lambda$ are the modulation wavelength, phase, and strength, respectively, which can be efficiently controlled via spatially structured substrates, as we demonstrate later. With these parameters, we are able to explore the full phase space of Coulomb pattern-induced topological effects. We solve this model via a mean-field decoupling yielding $H_{\mathrm{mf}}=\sum_{n, s} c_{n, s}^{\dagger} c_{n+1, s}+$ $\sum_{n} U_{n}\left\langle c_{n, s}^{\dagger} c_{n, s}\right\rangle c_{n, \bar{s}}^{\dagger} c_{n, \bar{s}}+$ H.c. Assuming time reversal symmetry, i.e., $\left\langle c_{n, \uparrow}^{\dagger} c_{n, \uparrow}\right\rangle=\left\langle c_{n, \downarrow}^{\dagger} c_{n, \downarrow}\right\rangle$, the interactions $U_{n}$ locally renormalize the onsite energies, following the modulation profile. This mean-field Hamiltonian is a realization of a diagonal Aubry-Andre-Harper model [37,38] for electrons with a nonzero charge average per site that is known to have edge states stemming from a parent two-dimensional (2D) Hall state $[39,40]$.

The topological invariant at certain excitation gaps of such models is given by the Chern number [39-43], which matches the number of states that cross the gap as a function of the phason $\phi[40,44]$. In Fig. 2(a), we show the bulk energy spectrum of a QD chain as a function of $\Omega$ at half-filling, which is clearly gapped in certain regions. As shown in Fig. 2(b), those gaps increase with $\tilde{U}$. These gaps are of topological origin [43,45], as depicted in Figs. 2(c) and 2(d), where we show the mean-field spectrum as a function of $\phi$. In particular, we see two edge modes (red and blue) that cross the gap as $\phi$ is changed. Spatially modulated onsite Hubbard interactions are thus indeed capable of generating topological nontrivial modes by modulating the effective single-particle mean-field Hamiltonian. This rather simple mechanism on the effective (a)
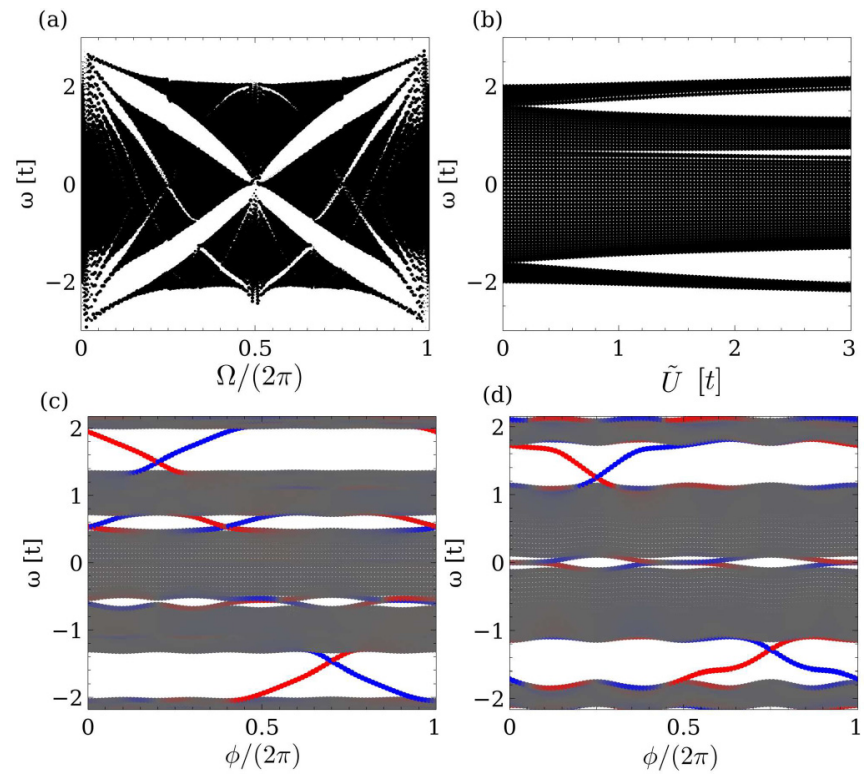

FIG. 2. Screening-induced mean-field topology. Bulk spectra as functions of (a) $\Omega$ and (b) $\tilde{U}$, showing the emergence of topological gaps. Full spectra as a function of $\phi$ for (c) $\Omega=0.4 \pi$ and (d) $\Omega=$ $0.5 \pi$, depicting edge states pumping through the gaps. Red (blue) denotes left (right) edge, the Fermi level is at $E=0.0$ and the system half filled. We use 80 sites and choose $\tilde{U}=6 t, \lambda=0.5$, and $\Omega=$ $0.4 \pi$ if not stated otherwise.

single-particle level exemplifies that interactions alone are able to induce nontrivial topology.

\section{SCREENING-INDUCED MANY-BODY TOPOLOGY}

In a more sophisticated scenario, we induce topological behavior in a regime with a topologically trivial mean-field Hamiltonian. To this end, we propose a similar interacting Hamiltonian as before but with exactly one electron per site:

$$
\begin{aligned}
H= & \sum_{n, s} t c_{n, s}^{\dagger} c_{n+1, s} \\
& +\sum_{n} U_{n}\left(c_{n, \uparrow}^{\dagger} c_{n, \uparrow}-\frac{1}{2}\right)\left(c_{n, \downarrow}^{\dagger} c_{n, \downarrow}-\frac{1}{2}\right)+\text { H.c. }
\end{aligned}
$$

This Hamiltonian can be understood as a Hubbard QD chain in which each QD is biased so that it is half filled. The corresponding mean-field decoupled Hamiltonian is by construction uniform $H_{\mathrm{mf}}=\sum_{n, s} t c_{n, s}^{\dagger} c_{n+1, s}+$ H.c. and thus topologically trivial. To exactly treat the Hamiltonian from Eq. 2, we use the tensor network formalism [46-52]. For all $\phi$, the system remains nonmagnetic and half filled in every site. We analyze the dynamical spin response defined by $\mathcal{S}(\omega, n)=\left\langle G S\left|S_{n}^{z} \delta\left(\omega-H+E_{G S}\right) S_{n}^{z}\right| G S\right\rangle$ that can be measured with inelastic spectroscopy [53] and which is shown in Figs. 3(a) and 3(c) as a function of $\phi$ for different $\Omega$. Again, we find edge excitations crossing bulk excitation gaps as the phason is changed. These finite spectral gaps are visible in the bulk spin responses shown in Figs. 3(b) and 3(d) and are present for arbitrary modulation frequencies $\Omega$, as depicted in Fig. 3(e), leading to in-gap edge excitations for generic $\Omega$. These gaps are furthermore proportional to the Hubbard 

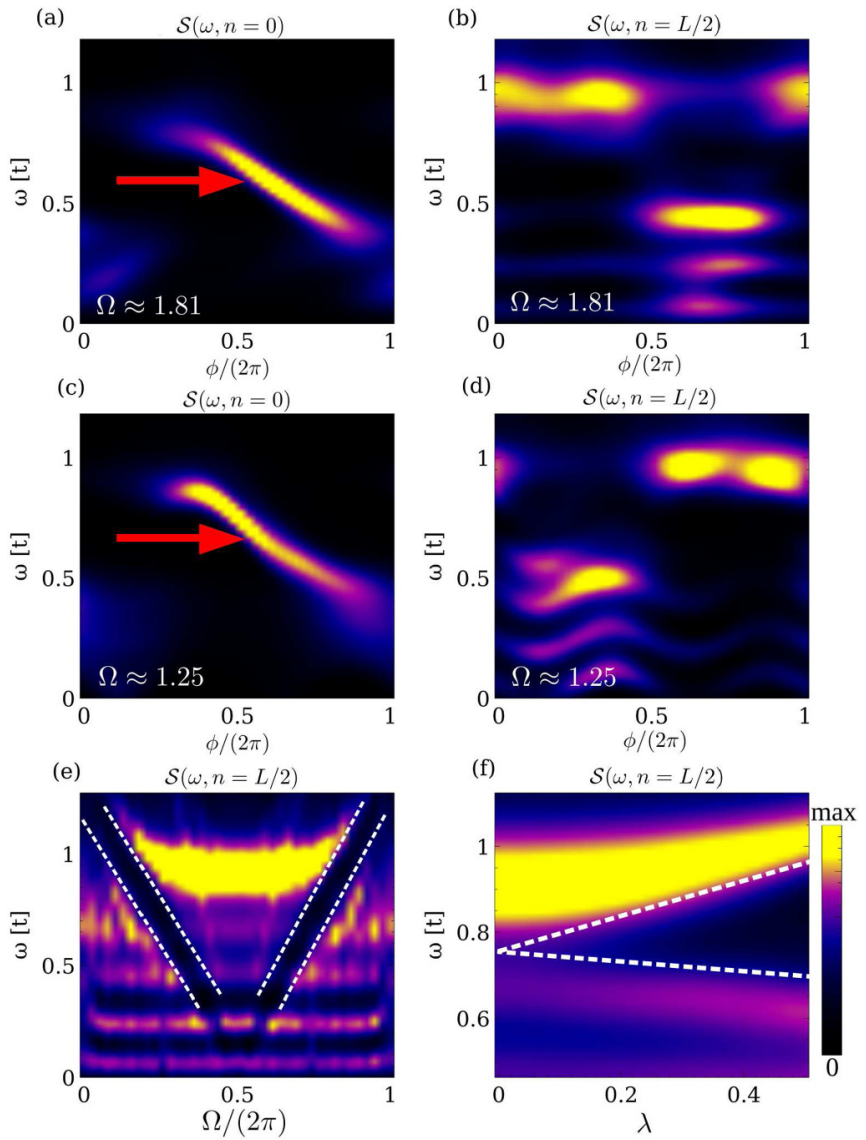

FIG. 3. Screening-induced many-body topology. Spin spectral function (a) in the edge and (b) in the bulk for $\Omega=\pi / \sqrt{3}$. Spectral function (c) in the edge and (d) in the bulk for $\Omega=\pi / 2$. (e) Bulk spectral function as a function of $\Omega$, showing gaps at generic values of $\Omega$. (f) Approximate linear scaling of the topological gap as a function of $\lambda$. We used 30 sites and set $\lambda=0.5, \tilde{U}=6 t$, and $\Omega=0.4 \pi$ if not stated otherwise.

modulation strength $\lambda$, as shown in Fig. 3(f). We verified that such in-gap modes are robust toward the presence of random disorder in the modulated local Coulomb interaction $U_{n}$ or interdot hopping $t^{\prime}$. These characteristics underline their topological origin $[40,44,54]$, which is here solely created by modulated many-body interactions.

The model of Eq. 2 is a many-body version of the AubryAndre-Harper model, as introduced in the previous section. However, a mapping to the parent electronic 2D quantum Hall state cannot be performed due to its genuine many-body nature. To understand how the engineered interactions are capable of creating topological edge modes here, we explore the model in the strong coupling limit, i.e., $\tilde{U} \gg t$. In this strongly interacting limit, spin and charge degrees of freedom are separated while every QD is still hosting one electron, giving rise to gaped charge excitations decoupled from the spin sector. This can be explicitly shown by performing a Schrieffer-Wolff transformation of the Hamiltonian from Eq. 2 (which is not possible for Eq. 1) into spin operators yielding the effective Hamiltonian

$$
H^{\mathrm{eff}}=\sum_{n} J_{n, n+1} \mathbf{S}_{n} \cdot \mathbf{S}_{n+1},
$$

where $\mathbf{S}_{n}$ are the $S=\frac{1}{2}$ operators on each site, and $J_{n, n+1}$ is the effective exchange interaction that takes the form $J_{n, n+1}=2 t^{2}\left(\frac{1}{U_{n}}+\frac{1}{U_{n+1}}\right) \approx \frac{4 t^{2}}{\tilde{U}}[1-\lambda \cos (\Omega n+\phi)][55]$. This Hamiltonian realizes a quasiperiodic antiferromagnetic $S=$ $1 / 2$ Heisenberg model, [56,57] whose ground state is a timereversal symmetric singlet state. Such a ground state is an entangled many-body state that cannot be described as a classical symmetry broken anti-ferromagnetic state due to strong quantum fluctuations. Its low-energy excitations have $S=\frac{1}{2}$, in contrast to $S=1$ of classical magnets. A common approach to characterize these low-energy excitations is to use a so-called parton Abrikosov fermion transformation of the form $S_{n}^{\alpha}=\sum_{s, s^{\prime}} \frac{1}{2} \sigma_{s, s^{\prime}}^{\alpha} f_{n, s}^{\dagger} f_{n, s}$, where $f_{n, s}^{\dagger}$ and $f_{n, s}$ are the creation and annihilation spinon operators [58]. Using this to transform the operators in $H^{\text {eff }}$ followed by a mean-field decoupling for the Abrokosov fermions, we obtain an effective Hamiltonian of the form $H_{P}^{\text {eff }}=\sum_{n, s} \gamma_{n, n+1} f_{n, s}^{\dagger} f_{n+1, s}+$ H.c. [59]. This effective Hamiltonian $H_{P}^{\text {eff }}$ describes fractionalized particles with $S=\frac{1}{2}$ and no charge, where the effective hoppings are proportional to the exchange coupling of the parent Heisenberg model, i.e., $\gamma_{n, n+1} \sim J_{n, n+1}$. Here, $H_{P}^{\text {eff }}$ thus again resembles an off-diagonal spinon Aubry-Andre-Harper model that can be mapped to a $2 \mathrm{D}$ quantum Hall state for spinons. As a result, the effective Hamiltonian $H^{\text {eff }}$ hosts topologically nontrivial edge spin excitations, and so does the original Hamiltonian from Eq. 2 in the strong-coupling limit, resulting from the spatially patterned local interactions. The topological behavior of the model from Eq. 2 is thus clearly induced by many-body effects alone and does not rely on any topological behavior on the single-particle or mean-field level.

Let us now briefly comment on the role of edge perturbations. A perturbation on the edge can change the energy of the edge mode discussed above, as in the single-particle Su-Schrieffer-Heeger (SSH) model [60-62], in second-order topological insulators [15,63-65], as well as in topological crystalline insulators $[10,66]$. In fact, the topologically nontrivial states of the SSH and higher-order topological models can be rationalized as highly specific examples of a generalized single-particle quasiperiodic topological mode [43]. In this regard, the topological origin of the modes in our manybody proposal share analogous robustness with the modes in the SSH [60-62] and higher-order models [15,63-65]. Finally, it is worth noting that, in the presence of an edge perturbation, the spectrum of the presented many-body models will always show an edge mode pumping the gap as the parameter $\phi$ is changed $[40,42,43,67]$.

\section{LOCAL COULOMB ENGINEERING}

After establishing the novel concept of Coulombengineered topology in one-dimensional (1D) systems, we now turn to the experimental feasibility. While 1D tightbinding-like chains have been already created and studied using metallic nanospheres [68,69], QDs [70,71], and even single atoms [72,73], spatially patterned Hubbard models have not been created yet. Thus, we will focus in the following on how a structuring of $U_{n}$ can be achieved using substratescreening effects. 
(a)
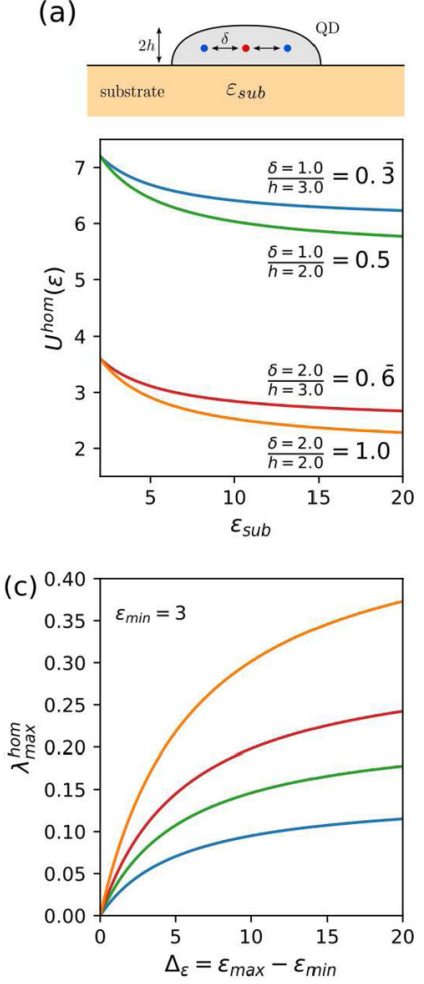

(b)
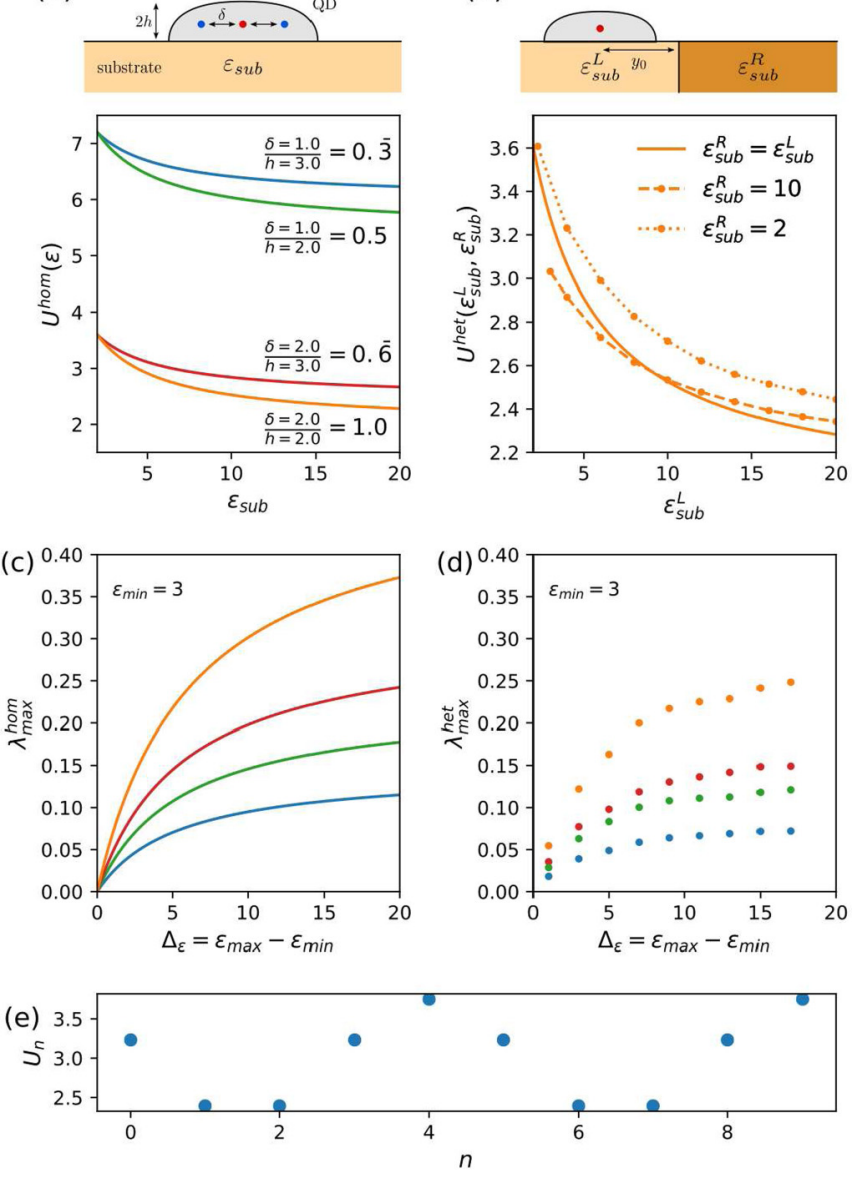

(f)

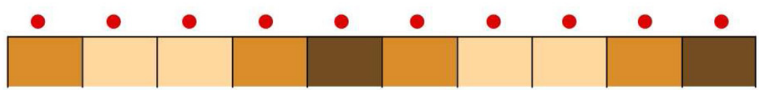

FIG. 4. Local Coulomb engineering. Local Coulomb interaction $U_{n}$ controlled by (a) homogeneous and (b) heterogeneous dielectric substrates. (c)-(d) Upper and lower limits of the Coulomb modulation strength $\lambda_{\max }$ estimated from homogeneous and heterogeneous substrates for different $\delta / h$ ratios and $\varepsilon_{\min }=3$. (e) Example $U_{n}$ profile corresponding to $\tilde{U}=3 \mathrm{eV}, \lambda=0.25$, and $\Omega=\phi=0.4 \pi$, which could be realized with a patterned substrate, as illustrated in the sketch (f).

The local Coulomb interactions used in the Hubbard models from above are matrix elements evaluated in a singleorbital Wannier basis $\psi(r)$ given by $U_{n}=\iint d r d r^{\prime} \mid \psi(r-$ $\left.r_{n}\right)\left.\right|^{2}\left|\psi\left(r^{\prime}-r_{n}\right)\right|^{2} U\left(r, r^{\prime}\right)$. In the following, we will approximate these elements by $U_{n} \approx e \Phi_{n}(\vec{\delta})$, where $\Phi_{n}(\vec{x})$ is the electrostatic potential of an electron with charge $e$ localized at the lattice position $n$ as felt by a second electron in its close vicinity (at distance $\vec{\delta}$ ), as depicted in Fig. 4(a). The screening effects of a homogeneous substrate can readily be calculated using the concept of image charges, as described in the Methods section. Here, $U_{n}^{\text {hom }}$ is then fully defined by the effective Wannier-orbital spread $\delta$ and the dot-substrate separation $h$, as depicted in Fig. 4(a) for different $\delta$ and $h$ as a function of the substrate screening constant $\varepsilon_{\text {sub }}$ and for a background $\varepsilon_{0}=2$. The unscreened (bare) value of the local Coulomb interaction is defined by $\delta$, whereas $h$ controls its vulnerability to $\varepsilon_{\text {sub }}$ [74]. We see that the local Coulomb interaction can be significantly modulated by changes to the homogeneous substrate screening.

The key element of our Coulomb-engineered topology proposal is a spatial modulation of the local interactions $U_{n}$. To achieve this, we propose to spatially structure the substrate screening. A corresponding heterogeneous setup with just one dielectric interface in the substrate is depicted in Fig. 4(b). Here, we approximate the resulting potential $\Phi(\vec{x})$ with a multi-image-charge ansatz to fulfill the necessary boundary conditions (see Methods for more details). Figure 4(b) shows a corresponding example in the form of $U^{\text {het }}\left(\varepsilon_{\text {sub }}^{L}, \varepsilon_{\text {sub }}^{R}\right)$ for $\delta / h=1$ [75]. We present data for $\varepsilon_{\text {sub }}^{R}=$ $\varepsilon_{\text {sub }}^{L}$ (homogeneous substrate), $\varepsilon_{\text {sub }}^{R}=2$, and $\varepsilon_{\text {sub }}^{R}=10$, from which we see that the homogeneous solution smoothly interpolates between the two heterogeneous situations for $\varepsilon_{\text {sub }}^{L} \in$ $[2,10]$. Here, $U^{\text {het }}\left(\varepsilon_{\text {sub }}^{L}, \varepsilon_{\text {sub }}^{R}=2\right)$ is always the largest due to the reduced screening from the right side of the substrate, while $U^{\text {het }}\left(\varepsilon_{\text {sub }}^{L}, \varepsilon_{\text {sub }}^{R}=10\right)$ is the smallest for $\varepsilon_{\text {sub }}^{L} \in[2,10]$. Most importantly, we find that there are just minor quantitative changes to the local Coulomb interaction screened by $\varepsilon_{\text {sub }}^{L}$ with $\varepsilon_{\text {sub }}^{R}$ being different in close vicinity. We can thus conclude that periodically patterned substrate screening functions with additional dielectric interfaces will not qualitatively affect the local substrate screening properties from the immediate surrounding.

As described above and shown in Fig. 3(f), the topological gap in the spin spectral function is proportional to $t^{2} \lambda / \tilde{U}$. The Coulomb modulation strength $\lambda$ thus plays a significant role for our proposal, as it maximizes the topological gap and thus protects the topological character of the system. To estimate the maximal possible modulation strength, we define $\lambda_{\max }=\frac{U_{\max }}{U_{\text {wa }}}-1=\frac{\Delta_{U}}{U_{\text {ave }}}$ with $\Delta_{U}=U_{\max }-U_{\min }$ and $U_{\text {ave }}=\left(U_{\max }+U_{\min }\right) / 2$, i.e., $\lambda_{\max }$ is defined by the the maximally and minimally achievable local interactions. For the homogeneous substrate, we can calculate $\lambda_{\max }^{\text {hom }}$ by defining $U_{\max }=U^{\text {hom }}\left(\varepsilon_{\min }\right)$ and $U_{\min }=U^{\text {hom }}\left(\varepsilon_{\max }\right)$ with $\varepsilon_{\min }<\varepsilon_{\max }$. In Fig. 4(c), we show the resulting values for fixed $\varepsilon_{\min }=$ 3 . Here, $\lambda_{\max }^{\text {hom }}$ steadily increases with the dielectric contrast $\Delta_{\varepsilon}$, which is driven by the enhancement of $\Delta_{U}$ (due to the reduction of $U_{\min }$ ) upon increasing $\varepsilon_{\max }$. Additionally, $\lambda_{\max }^{\text {hom }}$ increases with the $\delta / h$ ratio, which results from a decreased $U_{\text {ave }}$ for increased $\delta$ and the enhanced substrate-screening vulnerability of $U^{\text {hom }}$ upon decreasing $h$. Here, $\lambda_{\max }^{\text {hom }}$ is thus maximized by large dielectric contrasts and large $\delta / h$ ratios.

These homogeneous $\lambda_{\max }^{\text {hom }}$ are, however, just upper limits. In a more realistic setting, $U_{\max }$ and $U_{\min }$ might result from a heterogeneous substrate with additional dielectric interfaces, as depicted in the sketch of Figs. 4(e) and 4(f). To estimate $\lambda_{\max }^{\text {het }}$ in such a setting, we imagine the transition from $U_{\max }$ to $U_{\min }$ taking place within three lattice sites and set $U_{\max }=U^{\text {het }}\left(\varepsilon_{\min }, \varepsilon_{\text {mid }}\right)$ and $U_{\min }=U^{\text {het }}\left(\varepsilon_{\max }, \varepsilon_{\text {mid }}\right)$ with $\varepsilon_{\text {mid }}=\varepsilon_{\min }+\Delta_{\varepsilon} / 2$. The resulting values are shown in Fig. 4(d). Due to the additional dielectric interface in the substrate, $\Delta_{U}$ is decreased so that $\lambda_{\max }^{\text {het }}$ is overall smaller than $\lambda_{\max }^{\text {hom }}$, but behaves otherwise similar. For $\delta=2 \AA$ (orange and red dots) $\lambda_{\max }^{\text {het }}$ represents the lower limit since the interface is positioned here at $y_{0}=\delta=2 \AA$. By increasing $y_{0}, \lambda_{\max }^{\text {het }}$ approaches the upper limit $\lambda_{\max }^{\text {hom }}$. The optimal parameter regime to realize screening-induced many-body topology is thus defined by small QD heights $h$, large QD diameters $\delta$, large 
QD separations $y_{0}$, and large dielectric contrasts $\Delta_{\varepsilon}$, which increases $\lambda_{\max }$ and thus the topological gap.

\section{CONCLUSIONS}

We proposed a family of topological states whose topological excitations stem purely from many-body interactions instead of from single-particle engineering. Our proposal compares with conventional schemes that rely on engineering single-particle physics, demonstrating that engineered electronic interactions are a powerful complementary tool for exploring quantum states, which can be also used to create more complex models, such as second-order topological insulators $[15,43,64]$. Importantly, we showed that Coulomb engineering can be efficiently utilized to create the needed interaction profiles, demonstrating that our proposal can be implemented with current dielectric-engineering techniques. Our results thus put forward a method to create topological states of matter based on engineered interactions, providing a stepping stone to exploit dielectric engineering to realize exotic quantum excitations.

\section{ACKNOWLEDGMENTS}

We thank L. Muechler for fruitful discussions. J.L.L. acknowledges the computational resources provided by the Aalto Science-IT project and financial support from the Academy of Finland Projects No. 331342 and No. 336243.

\section{APPENDIX A: TENSOR NETWORK FORMALISM}

We solve exactly the many-body problems from the main text with the help of the kernel polynomial tensor network formalism [46-52]. Within the latter, we expand the spectral function in terms of Chebyshev polynomials, whose coefficients can be efficiently computed using a recursion relation between tensor network wave functions. Here, $\mathcal{S}(n, \omega)$ is subsequently represented in terms of $N$ Chebyshev polynomials $T_{k}(\omega)$ as $\mathcal{S}(\omega, n)=\frac{1}{\pi \sqrt{1-\omega^{2}}}\left[\mu_{0}+2 \sum_{k=1}^{N} \mu_{k} T_{k}(\omega)\right]$. The coefficients $\mu_{k}$ are defined by $\mu_{k}=\left\langle G S\left|S_{n}^{z} T_{k}(H) S_{n}^{z}\right| G S\right\rangle$, which can be efficiently computed using the Chebyshev recursion relations. Once the first $N / 2$ moments are computed, we use an autoregressive algorithm [76] to predict the next $N / 2$ moments and reconstruct the spectral function using a Jackson kernel [77]. The autoregressive model halves the calculation costs, and the Jackson kernel quenches Gibbs oscillations. This formalism allows us to compute dynamical response functions of the many-body system directly in frequency space, without requiring any time evolution.

\section{APPENDIX B: ROBUSTNESS OF THE EDGE MODES}

Here, we show how the topological edge modes are found to be robust against perturbations of the many-body Hamiltonian of Eq. 2. In particular, we explore two different perturbations that are especially relevant for the experimental realization: Second-neighbor hopping and disorder in the interactions. First, the nearest-neighbor hopping model of Eq. 2 is expected to be an approximation to the real system, as a finite overlap between second-neighbor sites
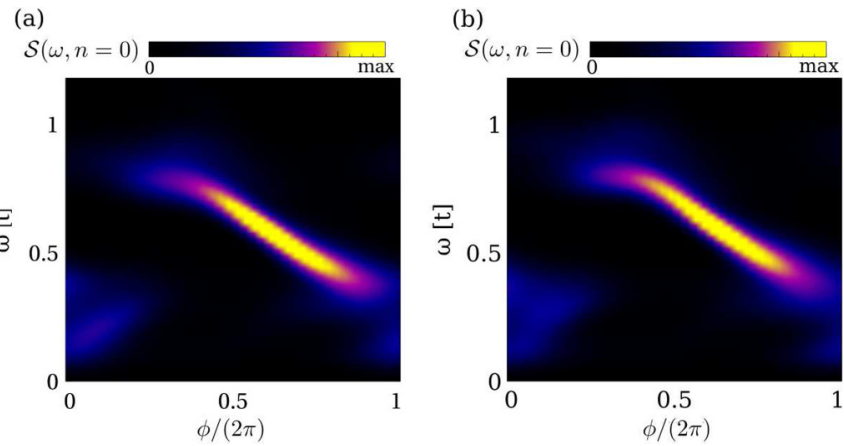

FIG. 5. Robustness of the edge modes: (a) Spectral function of the edge for a finite second-neighbor hopping, and (b) with a finite random disorder in the local interactions, showing that the topological edge modes survive those perturbations. We took $\tilde{U}=6 t$ and $\Omega=\pi / \sqrt{3}$.

is expected. We capture this by adding a perturbation of the form $H_{N N N}=t_{N N N} \sum_{n, s} c_{i, s}^{\dagger} c_{i+2, s}+$ H.c.. In Fig. 5(a), we show the spectral function at the edge under the influence of this additional perturbation for $t_{N N N}=0.1 t$, showing that the in-gap modes survive. Second, as our proposal requires us to engineer different dielectric environments for each dot, defects in the fabrication are expected to give rise to imperfect interaction profiles. This can be captured by adding to the Hamiltonian from Eq. 2 a term of the form $H_{W}=\sum_{n} W_{n}\left(c_{n, \uparrow}^{\dagger} c_{n, \uparrow}-\frac{1}{2}\right)\left(c_{n, \downarrow}^{\dagger} c_{n, \downarrow}-\frac{1}{2}\right)$ where $W_{n}$ are sitedependent random numbers. We show in Fig. 5(b) the spectral function at the edge, including this onsite Coulomb disorder for $W_{n} \in(-0.3 t, 0.3 t)$, showing that the in-gap edge modes again survive disorder. These results highlight the robustness of the edge modes toward perturbations that are likely to present in the experimental setup.

\section{APPENDIX C: POISSON SOLVER}

To calculate the substrate-screened local Coulomb interactions $U_{n}=e \Phi(\delta)$, we use the concept of image charges. In the case of a simple homogeneous substrate, we can choose an ansatz of the form

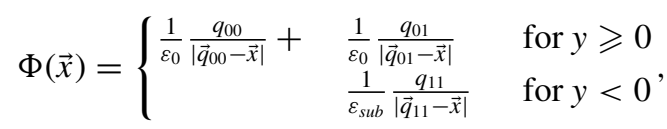

where $q_{00}$ and $\vec{q}_{00}$ are the charge and position of the source charge, and $q_{01}, q_{11}, \vec{q}_{01}$, and $\vec{q}_{11}$ are the charges and positions of the image charges. This potential needs to solve the Poisson equation in each $\varepsilon_{i}$ region, i.e.,

$$
\Delta \Phi(\vec{x})=\left\{\begin{array}{cc}
\frac{\rho(\vec{x})}{\varepsilon_{0}} & \text { for } y \geqslant 0 \\
0 & \text { for } y<0
\end{array},\right.
$$

where $\rho(\vec{x})$ is the point-charge density of the source charge $q_{00}$ and must fulfill the boundary conditions

$$
\begin{gathered}
\Phi\left(\vec{x}_{y^{+}}\right)=\Phi\left(\vec{x}_{y^{-}}\right), \\
\varepsilon_{0} \frac{\partial \Phi\left(\vec{x}_{y^{+}}\right)}{\partial y}=\varepsilon_{\mathrm{sub}} \frac{\partial \Phi\left(\vec{x}_{y^{-}}\right)}{\partial y}
\end{gathered}
$$



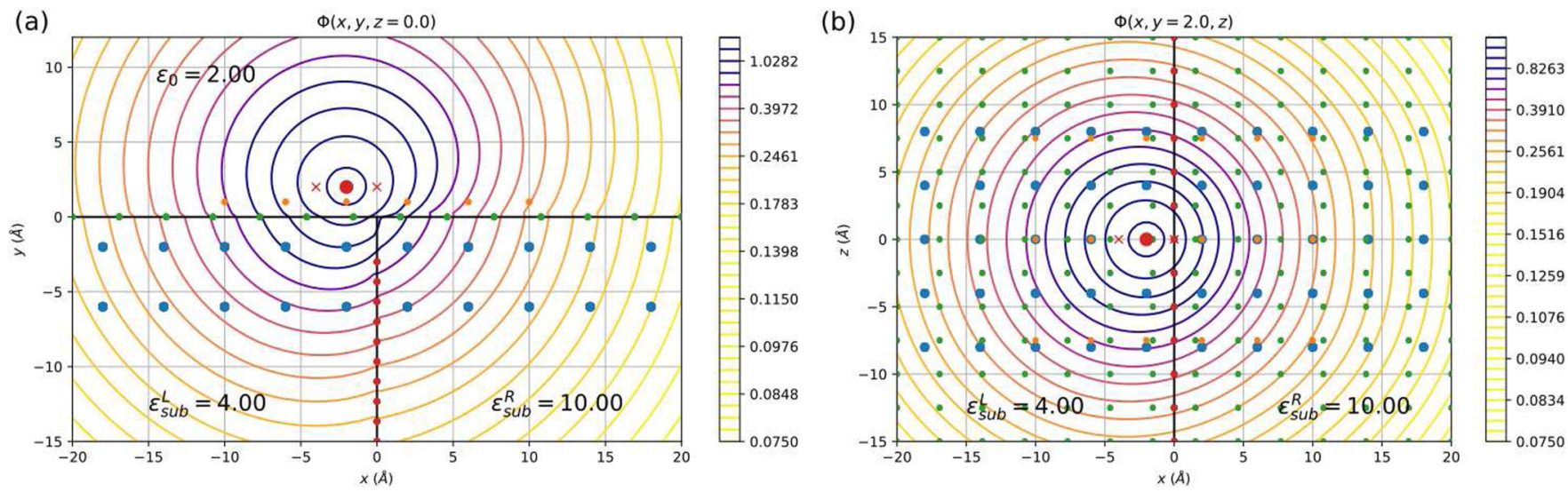

FIG. 6. Example result of the multi-image-charge approach. (a) Left and (b) right show the numerically evaluated potential for $\varepsilon_{0}=2$, $\varepsilon_{\text {sub }}^{L}=4, \varepsilon_{\text {sub }}^{R}=10, h=2 \AA$, and $\delta=y_{0}=2 \AA$ in the $(x, y)$ and $(x, z)$ planes. Large red dot: Source charge position, orange dots: Poisson equation evaluation points, green dots: $y=0$ interface discrete boundary condition points, red dots: $x=0$ interface discrete boundary condition points, blue dots: Image charge positions, black lines: Dielectric interfaces.

at the dielectric interface defined by $y=0$. By exploiting the full rotational symmetry around the $y$ axis (through the source charge), we can readily fix $\vec{q}_{01}$ to the $y=0$ plane mirrored position of $\vec{q}_{00}$ and choose $\vec{q}_{11}=\vec{q}_{00}$. Subsequently, $q_{01}$ and $q_{11}$ are fixed by the boundary conditions yielding

$$
\begin{gathered}
q_{01}=\frac{\varepsilon_{0}-\varepsilon_{\text {sub }}}{\varepsilon_{0}+\varepsilon_{\text {sub }}} q_{00}, \\
q_{11}=q_{01}-q_{00} .
\end{gathered}
$$

Thus, in the case of a homogeneous substrate, the influence of $\varepsilon_{\text {sub }}$ to the local Coulomb interaction $U_{n}$ can be calculated analytically. As soon as there is an additional dielectric interface in the substrate, we cannot find an analytic solution anymore. To estimate the impact of this heterogeneous substrate screening, we construct an approximate solution from a multiple image charge ansatz of the form

$$
\Phi(\vec{x})=\left\{\begin{array}{lll}
\frac{1}{\varepsilon_{0}} \frac{q_{00}}{\left|\vec{q}_{00}-\vec{x}\right|}+ & \frac{1}{\varepsilon_{0}} \sum_{i}^{2 N} \frac{q_{0 i}}{\left|\vec{q}_{0 i}-\vec{x}\right|}, & y \geqslant 0 \\
& \frac{1}{\varepsilon_{\text {sub }}^{L}} \sum_{i}^{N} \frac{q_{1 i}}{\left|\vec{q}_{1 i}-\vec{x}\right|}, & y<0, x \leqslant 0, \\
& \frac{1}{\varepsilon_{\text {sub }}^{R}} \sum_{i}^{N} \frac{q_{2 i}}{\left|\vec{q}_{2 i}-\vec{x}\right|}, & y<0, x>0
\end{array}\right.
$$

which is supposed to solve the Poisson equation given in Eq. (C2) with the boundary conditions at the $y=0$ interfaces

$$
\begin{aligned}
& \Phi\left(\vec{x}_{y^{+}}\right)=\Phi\left(\vec{x}_{y^{-}}\right), \quad y=0, \\
& \varepsilon_{0} \frac{\partial \Phi\left(\vec{x}_{y^{+}}\right)}{\partial y}=\varepsilon_{\text {sub }}^{L} \frac{\partial \Phi\left(\vec{x}_{y^{-}}\right)}{\partial y}, \quad y=0, x \leqslant 0, \\
& \varepsilon_{0} \frac{\partial \Phi\left(\vec{x}_{y^{+}}\right)}{\partial y}=\varepsilon_{\text {sub }}^{R} \frac{\partial \Phi\left(\vec{x}_{y^{-}}\right)}{\partial y}, \quad y=0, x>0,
\end{aligned}
$$

and at the $x=0$ interface in the substrate

$$
\begin{gathered}
\Phi\left(\vec{x}_{x^{+}}\right)=\Phi\left(\vec{x}_{x^{-}}\right), \quad y<0, x=0, \\
\varepsilon_{\text {sub }}^{L} \frac{\partial \Phi\left(\vec{x}_{x^{+}}\right)}{\partial x}=\varepsilon_{\text {sub }}^{R} \frac{\partial \Phi\left(\vec{x}_{x^{-}}\right)}{\partial x}, \quad y<0, x=0 .
\end{gathered}
$$

If we fix all image charge positions, we can use the $4 N$ image charges $q_{(0,1,2) i}$ to fulfill $4 N$ boundary conditions at discrete interface positions $\vec{x}_{i}$. The resulting linear equation system is well defined and has a unique solution. Here, however, we reformulate the Poisson equation into a minimization problem of the form

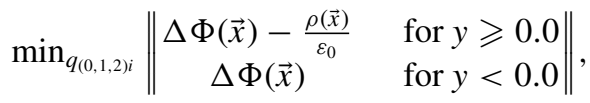

using the boundary conditions from Eqs. ((C8)-(C12)) to define constraints for the minimization. This relaxes the oneto-one correspondence between the number of image charges and the number of discrete boundary conditions.

Numerically, we use the Sequential Least SQuares Programming (SLSQP) algorithm [78] as implemented in the Scipy minimization package. We use $5 \times 2 \times 5$ image charges per substrate region (i.e., 100 in total), separated by $\left(d_{x}, d_{y}, d_{z}\right)=(4,4,4) \AA$ and distributed, as indicated in Fig. 6 , and 182 boundary condition points at the $y=0$ interface and 130 points at the $x=0$ interface distributed, as also indicated in Fig. 6. To minimize the function defined in Eq. (C13), we evaluate the Poisson equation on a discrete grid of 18 points in the $x-z$ plane slightly below the source charge (see Fig. 6). The source charge is positioned at $\vec{q}_{00}=\left(h, y_{0}=-2,0\right)$ (with variable distance $h$ to the $y=0$ interface and fixed position relative to the vertical interface in the substrate), and its charge distribution is approximated using a three-dimensional Gauss function of the form

$$
\rho(\vec{x})=\frac{q_{00}}{\sigma^{3}(2 \pi)^{3 / 2}} \exp \left[-\frac{\left(\vec{q}_{00}-\vec{x}\right)^{2}}{\sigma^{2}}\right],
$$

with $\sigma=0.15 \AA$. Finally, $U_{n}=e \Phi(\vec{\delta})$ is evaluated using $e q_{00} \approx 14.39 \mathrm{eV} \AA$ and $\vec{\delta}=\vec{q}_{00}+(\delta, 0,0)$ with variable $\delta$.

In Fig. 6, we show a corresponding example result for $\varepsilon_{0}=$ $2, \varepsilon_{\text {sub }}^{L}=4, \varepsilon_{\text {sub }}^{R}=10, h=2 \AA$, and $\delta=\left|y_{0}\right|=2 \AA$. In this situation, we would a priori expect to see equipotential lines with small kinks at the $\varepsilon_{0} / \varepsilon_{\text {sub }}^{L}$ boundary and with enhanced kinks at the interfaces to the $\varepsilon_{\text {sub }}^{R}$ area, which is approximately the case in our numerical estimate. Furthermore, we see that these boundary conditions are also approximately fulfilled between the discrete boundary positions in the vicinity of the 
source charge (large red dot). Further away, we however also find deviations to this. We thus expect our numerical solution to be approximately valid in the vicinity of the source charge, which is enough to estimate the local Coulomb interaction.
[1] X.-L. Qi and S.-C. Zhang, Topological insulators and superconductors, Rev. Mod. Phys. 83, 1057 (2011).

[2] M. Z. Hasan and C. L. Kane, Colloquium: Topological insulators, Rev. Mod. Phys. 82, 3045 (2010).

[3] Y. Ando, Topological insulator materials, J. Phys. Soc. Jpn. 82, 102001 (2013).

[4] C. L. Kane and E. J. Mele, Quantum Spin Hall Effect in Graphene, Phys. Rev. Lett. 95, 226801 (2005).

[5] F. D. M. Haldane, Model for a Quantum Hall Effect without Landau Levels: Condensed-Matter Realization of the "Parity Anomaly”, Phys. Rev. Lett. 61, 2015 (1988).

[6] C.-Z. Chang, J. Zhang, X. Feng, J. Shen, Z. Zhang, M. Guo, K. Li, Y. Ou, P. Wei, L.-L. Wang, Z.-Q. Ji, Y. Feng, S. Ji, X. Chen, J. Jia, X. Dai, Z. Fang, S.-C. Zhang, K. He, Y. Wang et al., Experimental observation of the quantum anomalous hall effect in a magnetic topological insulator, Science 340, 167 (2013).

[7] C. W. J. Beenakker, Search for Majorana fermions in superconductors, Annu. Rev. Condens. Matter Phys. 4, 113 (2013).

[8] V. Mourik, K. Zuo, S. M. Frolov, S. R. Plissard, E. P. A. M. Bakkers, and L. P. Kouwenhoven, Signatures of majorana fermions in hybrid superconductor-semiconductor nanowire devices, Science 336, 1003 (2012).

[9] S. Nadj-Perge, I. K. Drozdov, J. Li, H. Chen, S. Jeon, J. Seo, A. H. MacDonald, B. A. Bernevig, and A. Yazdani, Observation of Majorana fermions in ferromagnetic atomic chains on a superconductor, Science 346, 602 (2014).

[10] L. Fu, Topological Crystalline Insulators, Phys. Rev. Lett. 106, 106802 (2011).

[11] Y. Tanaka, Z. Ren, T. Sato, K. Nakayama, S. Souma, T. Takahashi, K. Segawa, and Y. Ando, Experimental realization of a topological crystalline insulator in SnTe, Nat. Phys. 8, 800 (2012).

[12] J. Seidel, Nanoelectronics based on topological structures, Nat. Mater. 18, 188 (2019).

[13] L. Šmejkal, Y. Mokrousov, B. Yan, and A. H. MacDonald, Topological antiferromagnetic spintronics, Nat. Phys. 14, 242 (2018).

[14] J. Alicea, Y. Oreg, G. Refael, F. von Oppen, and M. P. A. Fisher, Non-abelian statistics and topological quantum information processing in 1D wire networks, Nat. Phys. 7, 412 (2011).

[15] F. Schindler, A. M. Cook, M. G. Vergniory, Z. Wang, S. S. P. Parkin, B. A. Bernevig, and T. Neupert, Higher-order topological insulators, Sci. Adv. 4, eaat0346 (2018).

[16] F. Schindler, Z. Wang, M. G. Vergniory, A. M. Cook, A. Murani, S. Sengupta, A. Yu. Kasumov, R. Deblock, S. Jeon, I. Drozdov, H. Bouchiat, S. Guéron, A. Yazdani, B. A. Bernevig, and T. Neupert, Higher-order topology in bismuth, Nat. Phys. 14, 918 (2018).

[17] Z. Gong, Y. Ashida, K. Kawabata, K. Takasan, S. Higashikawa, and M. Ueda, Topological Phases of Non-Hermitian Systems, Phys. Rev. X 8, 031079 (2018).
[18] H. Shen, B. Zhen, and L. Fu, Topological Band Theory for NonHermitian Hamiltonians, Phys. Rev. Lett. 120, 146402 (2018).

[19] M. M. Denner, A. Skurativska, F. Schindler, M. H. Fischer, R Thomale, T. Bzdušek, and T. Neupert, Exceptional topological insulators, arXiv:2008.01090 (2020).

[20] H. C. Po, H. Watanabe, and A. Vishwanath, Fragile Topology and Wannier Obstructions, Phys. Rev. Lett. 121, 126402 (2018).

[21] J. Ahn, S. Park, and B.-J. Yang, Failure of Nielsen-Ninomiya Theorem and Fragile Topology in Two-Dimensional Systems with Space-Time Inversion Symmetry: Application to Twisted Bilayer Graphene at Magic Angle, Phys. Rev. X 9, 021013 (2019).

[22] Y. E. Kraus and O. Zilberberg, Quasiperiodicity and topology transcend dimensions, Nat. Phys. 12, 624 (2016).

[23] Y. E. Kraus, Y. Lahini, Z. Ringel, M. Verbin, and O. Zilberberg, Topological States and Adiabatic Pumping in Quasicrystals, Phys. Rev. Lett. 109, 106402 (2012).

[24] A. Agarwala and V. B. Shenoy, Topological Insulators in Amorphous Systems, Phys. Rev. Lett. 118, 236402 (2017).

[25] K. Pöyhönen, I. Sahlberg, A. Westström, and T. Ojanen, Amorphous topological superconductivity in a Shiba glass, Nat. Commun. 9, 2103 (2018).

[26] E. Dagotto, Correlated electrons in high-temperature superconductors, Rev. Mod. Phys. 66, 763 (1994).

[27] J. K. Jain, Composite-Fermion Approach for the Fractional Quantum Hall Effect, Phys. Rev. Lett. 63, 199 (1989).

[28] R. Willett, J. P. Eisenstein, H. L. Störmer, D. C. Tsui, A. C. Gossard, and J. H. English, Observation of an EvenDenominator Quantum Number in the Fractional Quantum Hall Effect, Phys. Rev. Lett. 59, 1776 (1987).

[29] S. Raghu, X.-L. Qi, C. Honerkamp, and S.-C. Zhang, Topological Mott Insulators, Phys. Rev. Lett. 100, 156401 (2008).

[30] M. Dzero, K. Sun, V. Galitski, and P. Coleman, Topological Kondo Insulators, Phys. Rev. Lett. 104, 106408 (2010).

[31] M. Dzero, J. Xia, V. Galitski, and P. Coleman, Topological Kondo insulators, Annu. Rev. Condens. Matter Phys. 7, 249 (2016).

[32] W. Chen and J. L. Lado, Interaction-Driven Surface Chern Insulator in Nodal Line Semimetals, Phys. Rev. Lett. 122, 016803 (2019).

[33] M. Rösner, C. Steinke, M. Lorke, C. Gies, F. Jahnke, and T. O. Wehling, Two-Dimensional Heterojunctions from Nonlocal Manipulations of the Interactions, Nano Lett. 16, 2322 (2016).

[34] A. Raja, A. Chaves, J. Yu, G. Arefe, H. M. Hill, A. F. Rigosi, T. C. Berkelbach, P. Nagler, C. Schüller, T. Korn, C. Nuckolls, J. Hone, L. E. Brus, T. F. Heinz, D. R. Reichman, and A. Chernikov, Coulomb engineering of the bandgap and excitons in two-dimensional materials, Nat. Commun. 8, 15251 (2017).

[35] M. I. B. Utama, H. Kleemann, W. Zhao, C. Shen Ong, F. H. da Jornada, D. Y. Qiu, H. Cai, H. Li, R. Kou, S. Zhao, S. Wang, K. Watanabe, T. Taniguchi, S. Tongay, A. Zettl, S. G. Louie, 
and F. Wang, A dielectric-defined lateral heterojunction in a monolayer semiconductor, Nat. Electronics 2, 60 (2019).

[36] L. Waldecker, A. Raja, M. Rösner, C. Steinke, A. Bostwick, R. J. Koch, C. Jozwiak, T. Taniguchi, K. Watanabe, E. Rotenberg, T. O. Wehling, and T. F. Heinz, Rigid Band Shifts in Two-Dimensional Semiconductors through External Dielectric Screening, Phys. Rev. Lett. 123, 206403 (2019).

[37] P. G. Harper, Single band motion of conduction electrons in a uniform magnetic field, Proc. Phys. Soc., Sect. A 68, 874 (1955).

[38] S. Aubry and G. André, Analyticity breaking and Anderson localization in incommensurate lattices, Phys. Soc. 3, 18 (1980).

[39] D. J. Thouless, M. Kohmoto, M. P. Nightingale, and M. den Nijs, Quantized Hall Conductance in a Two-Dimensional Periodic Potential, Phys. Rev. Lett. 49, 405 (1982).

[40] D. J. Thouless, Quantization of particle transport, Phys. Rev. B 27, 6083 (1983).

[41] J. Kellendonk, Noncommutative geometry of tilings and gap labelling, Rev. Math. Phys. 07, 1133 (1995).

[42] O. Zilberberg, Topology in quasicrystals, arXiv:2012.03644 (2020).

[43] I. Petrides and O. Zilberberg, Higher-order topological insulators, topological pumps and the quantum Hall effect in high dimensions, Phys. Rev. Research 2, 022049(R) (2020).

[44] Y. E. Kraus and O. Zilberberg, Topological Equivalence between the Fibonacci Quasicrystal and the Harper Model, Phys. Rev. Lett. 109, 116404 (2012).

[45] Z.-W. Zuo, D. wei Kang, and L. Li, Topological end states in a one-dimensional spatially modulated interaction spinless fermion model, New J. Phys. 22, 083057 (2020).

[46] A. Weiße, G. Wellein, A. Alvermann, and H. Fehske, The kernel polynomial method, Rev. Mod. Phys. 78, 275 (2006).

[47] F. A. Wolf, J. A. Justiniano, I. P. McCulloch, and U. Schollwöck, Spectral functions and time evolution from the Chebyshev recursion, Phys. Rev. B 91, 115144 (2015).

[48] V. Kaskela and J. L. Lado, Dynamical topological excitations in parafermion chains, Phys. Rev. Research 3, 013095 (2021).

[49] J. L. Lado and M. Sigrist, Solitonic in-gap modes in a superconductor-quantum antiferromagnet interface, Phys. Rev. Research 2, 023347 (2020).

[50] ITensor Library, http://itensor.org.

[51] M. Fishman, S. R. White, and E. Miles Stoudenmire, The ITensor software library for tensor network calculations, arXiv:2007.14822 (2020).

[52] DMRGpy Library, https://github.com/joselado/dmrgpy.

[53] A. Spinelli, B. Bryant, F. Delgado, J. Fernández-Rossier, and A. F. Otte, Imaging of spin waves in atomically designed nanomagnets, Nat. Mater. 13, 782 (2014).

[54] M. Lohse, C. Schweizer, H. M. Price, O. Zilberberg, and I. Bloch, Exploring 4D quantum Hall physics with a 2D topological charge pump, Nature (London) 553, 55 (2018).

[55] We take $\lambda \ll 1$ and $\Omega \ll 1$.

[56] J. L. Lado and O. Zilberberg, Topological spin excitations in Harper-Heisenberg spin chains, Phys. Rev. Research 1, 033009 (2019).

[57] U. Agrawal, S. Gopalakrishnan, and R. Vasseur, Universality and quantum criticality in quasiperiodic spin chains, Nat. Commun. 11, 2225 (2020).
[58] L. Savary and L. Balents, Quantum spin liquids: A review, Rep. Prog. Phys. 80, 016502 (2016).

[59] We assume in the mean-field ansatz that time reversal symmetry is not broken and that no anomalous terms are generated, as will be expected for the uniform Heisenberg model.

[60] W. P. Su, J. R. Schrieffer, and A. J. Heeger, Solitons in Polyacetylene, Phys. Rev. Lett. 42, 1698 (1979).

[61] R. Drost, T. Ojanen, A. Harju, and P. Liljeroth, Topological states in engineered atomic lattices, Nat. Phys. 13, 668 (2017).

[62] O. Gröning, S. Wang, X. Yao, C. A. Pignedoli, G. B. Barin, C. Daniels, A. Cupo, V. Meunier, X. Feng, A. Narita, K. Müllen, P. Ruffieux, and R. Fasel, Engineering of robust topological quantum phases in graphene nanoribbons, Nature (London) 560, 209 (2018).

[63] W. A. Benalcazar, B. A. Bernevig, and T. L. Hughes, Electric multipole moments, topological multipole moment pumping, and chiral hinge states in crystalline insulators, Phys. Rev. B 96, 245115 (2017).

[64] W. A. Benalcazar, B. A. Bernevig, and T. L. Hughes, Quantized electric multipole insulators, Science 357, 61 (2017).

[65] S. N. Kempkes, M. R. Slot, J. J. van den Broeke, P. Capiod, W. A. Benalcazar, D. Vanmaekelbergh, D. Bercioux, I. Swart, and C. M. Smith, Robust zero-energy modes in an electronic higher-order topological insulator, Nat. Mater. 18, 1292 (2019).

[66] S. E. Freeney, J. J. van den Broeke, A. J. J. Harsveld van der Veen, I. Swart, and C. Morais Smith, Edge-Dependent Topology in Kekulé Lattices, Phys. Rev. Lett. 124, 236404 (2020).

[67] H. M. Price, O. Zilberberg, T. Ozawa, I. Carusotto, and N. Goldman, Four-Dimensional Quantum Hall Effect with Ultracold Atoms, Phys. Rev. Lett. 115, 195303 (2015).

[68] J. R. Krenn, A. Dereux, J. C. Weeber, E. Bourillot, Y. Lacroute, J. P. Goudonnet, G. Schider, W. Gotschy, A. Leitner, F. R. Aussenegg, and C. Girard, Squeezing the Optical Near-Field Zone by Plasmon Coupling of Metallic Nanoparticles, Phys. Rev. Lett. 82, 2590 (1999).

[69] S. A. Maier, M. L. Brongersma, P. G. Kik, and H. A. Atwater, Observation of near-field coupling in metal nanoparticle chains using far-field polarization spectroscopy, Phys. Rev. B 65, 193408 (2002).

[70] J. H. Lee, Z. M. Wang, B. L. Liang, W. T. Black, V. P. Kunets, Y. I. Mazur, and G. J. Salamo, Selective growth of InGaAs/GaAs quantum dot chains on pre-patterned GaAs(100), Nanotechnology 17, 2275 (2006).

[71] V. P. Kunets, M. Rebello Sousa Dias, T. Rembert, M. E. Ware, Yu. I. Mazur, V. Lopez-Richard, H. A. Mantooth, G. E. Marques, and G. J. Salamo, Electron transport in quantum dot chains: Dimensionality effects and hopping conductance, J. Appl. Phys. 113, 183709 (2013).

[72] H. Kim, A. Palacio-Morales, T. Posske, L. Rózsa, K. Palotás, L. Szunyogh, M. Thorwart, and R. Wiesendanger, Toward tailoring Majorana bound states in artificially constructed magnetic atom chains on elemental superconductors, Sci. Adv. 4, eaar5251 (2018).

[73] A. Kamlapure, L. Cornils, J. Wiebe, and R. Wiesendanger, Engineering the spin couplings in atomically crafted spin chains on an elemental superconductor, Nat. Commun. 9, 3253 (2018).

[74] Systems with a small Wannier-orbital spread $\delta$ have large local Coulomb interactions, and systems with small effective heights $h$ are more strongly affected by the substrate. 
[75] We assume the location of the source charge above $\varepsilon_{\text {sub }}^{L}$, as also depicted in the sketch of Fig. 4(b).

[76] H. Akaike, Fitting autoregressive models for prediction, Ann. Inst. Stat. Math. 21, 243 (1969).
[77] D. Jackson, On approximation by trigonometric sums and polynomials, Trans. Am. Math. Soc. 13, 491 (1912).

[78] D. Kraft, A Software Package for Sequential Quadratic Programming, Tech. Rep. (Institute for Flight Mechanics, Köln, 1988). 